\title{
Identification and characterization of DNA aptamers specific to VP2 protein of canine parvovirus
}

\author{
Mithilesh Singh ${ }^{1} \cdot$ Pranav Tripathi $^{2} \cdot$ Smriti Singh $^{2} \cdot$ Manisha Sachan $^{2} \cdot$ Vishal Chander $^{3} \cdot$ Gaurav Kumar Sharma $^{3}$. \\ Ujjwal Kumar De ${ }^{4}$ Sathish Kota ${ }^{5} \cdot$ Kalyani Putty $^{6} \cdot$ Raj Kumar Singh ${ }^{4} \cdot$ Seema Nara $^{2} \mathbb{C}$
}

Received: 21 May 2021 / Revised: 13 October 2021 / Accepted: 15 October 2021 / Published online: 29 October 2021

(c) The Author(s), under exclusive licence to Springer-Verlag GmbH Germany, part of Springer Nature 2021

\begin{abstract}
Canine parvovirus-2 (CPV-2) is ubiquitously distributed in dog population worldwide causing a severe and often fatal gastroenteritis. Owing to its highly contagious nature, rapid detection of CPV is crucial in effective control of the disease. Aptamers have emerged as potential alternative to antibodies as affinity reagents in diagnostic field. Present study was aimed to select and validate ssDNA aptamers specific to CPV. Systematic evolution of ligands through exponential enrichment (SELEX) method was employed for selection of CPV structural protein (VP2) specific DNA aptamers. SELEX was performed using a pool of ssDNA library comprising 30 random nucleotide region. A total of seven rounds of SELEX were performed using VP2 protein as target antigen which yielded ten aptamers (1A-10A) with distinct sequences. Target binding of all ten aptamers was assessed by dot blot and enzyme-linked oligonucleotide assay (ELONA) which revealed that 5A, 6A, 9A, and 10A were superior binders. In silico analysis of the aptamers revealed the existence of binding site on VP2 protein, and binding pattern was similar to in vitro findings. The affinity $\left(K_{D}\right)$ of all these four binders against CPV was evaluated by ELONA indicating relatively higher affinity of $6 \mathrm{~A}$ and $10 \mathrm{~A}$ than remaining two DNA sequences. Out of which, aptamer 6A displayed cross-reactivity with canine distemper virus and canine corona virus. Hence, aptamer 10A was considered as better binding sequence having high specificity and affinity for CPV. The study confirms the future utility of selected aptamers in development of a reliable diagnostic for rapid detection of CPV.
\end{abstract}

\section{Key points}

- Canine parvovirus-specific ssDNA aptamers were identified with nanomolar affinity (100-150 nM).

- Three aptamers displayed negligible cross-reactivity with other related viruses.

- Aptamer 10A displayed high binding affinity and specificity to CPV.

Keywords CPV $\cdot$ VP2 $\cdot$ Aptamer $\cdot$ ELONA

Mithilesh Singh and Pranav Tripathi These authors contributed equally to the work and are joint first authors

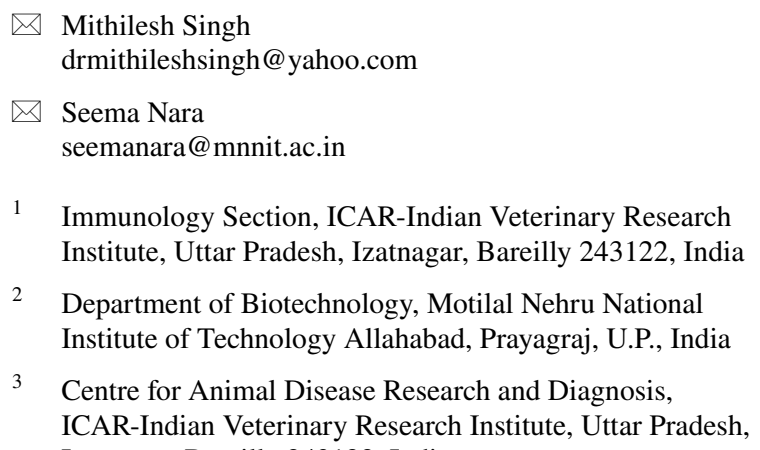

2 Department of Biotechnology, Motilal Nehru National Institute of Technology Allahabad, Prayagraj, U.P., India

3 Centre for Animal Disease Research and Diagnosis, ICAR-Indian Veterinary Research Institute, Uttar Pradesh, 


\section{Introduction}

Canine parvoviral enteritis remains to be one of the most dreaded viral diseases of domestic and wild canids worldwide (Chander et al. 2016). Acute enteritis is the disease's most severe clinical form mainly characterized by vomiting and hemorrhagic gastroenteritis in dogs of all ages, but more pronounced in pups (Eregowda et al. 2020). The clinical signs of CPV infection are non-specific and identical to those seen in other infections of gastrointestinal tract. Despite a regular practice of active immunization, CPV remains a worldwide threat to the lives of canines (Kelman et al. 2020). CPV-2 is a minute, negative-sense, non-enveloped single-stranded DNA virus distinct from $\mathrm{CPV}-1$ as later is considered non-pathogenic to dogs. CPV genome encodes two structural (VP1, VP2) and two nonstructural (NS1, NS2) proteins. The major structural or capsid protein is VP2 that plays a key role in determining antigenic properties, host range, and tissue tropism ( $\mathrm{Qi}$ et al. 2020). Original CPV-2 has three main antigenic variants, viz., CPV-2a, CPV-2b, and CPV-2c reported across the world.

Owing to highly contagious nature of the canine parvoviral infections, rapid diagnosis is essential in order to begin appropriate treatment as well as quarantine of infected dogs. Currently, several methods are available to detect canine parvovirus (CPV), each having individual benefits and disadvantages which should be considered in their application. The major limitations with most of these tests include requirement of specialized facilities/equipment or laboratories and thus not applicable for field use. At present, lateral flow immunoassay (LFIA) is the most commonly used test for the diagnosis of CPV infection in dogs because of simplicity and flexibility in its use. Test is quite rapid, as results can be visualized within a few minutes (Sharma et al. 2018). However, there are still issues of sensitivity, specificity, and its high cost in comparison to other tests (Decaro and Buonavoglia 2012). Additionally, other concerns may be related to the ability of LFIA to detect reported new antigenic variants of CPV-2 (new $\mathrm{CPV} 2 \mathrm{a} / \mathrm{CPV}-2 \mathrm{~b}$ and other variants with novel mutations) with the same accuracy as original CPV-2a/2b/2c. Besides this, most of the available commercial lateral flow assay (LFA) developed so far for detection of CPV uses antibodies as capture reagents and hence encounter various drawbacks associated with the use of antibodies. Hence, currently available diagnostic assays for CPV detection need refinement for regular and large-scale use.

Aptamers have emerged as good alternative to antibodies as affinity reagents and find applications in diagnostics, molecular, and environmental sector (Tombelli et al. 2007; Liu et al. 2009; Torres-Chavolla and Alocilja 2009).
Single-stranded oligonucleotide (both DNA and RNA) aptamers offer some inherent advantages over antibodies such as high affinity and specificity, stability, resistance to different chemical buffers, wide selection opportunities from a large library, easy synthesis, consistency in sequence between different batches, cost effectiveness, easy surface modification, and bioconjugation abilities (Radi 2011; Joshi et al. 2009). Further, aptamer synthesis does not require use of animals, a significant advantage considering the existing stringent animal ethics regulations. The potential of aptamers as affinity binder and as an efficient diagnostic tool has been explored for viruses such as influenza virus (Hmila et al. 2017), Newcastle disease virus (Marnissi et al. 2020), and bovine parainfluenza virus (Cheng et al. 2018). However, to date, there are no reports on identifying aptamers against CPV.

Thus, the present study reports the screening and identification of specific ssDNA aptamers against recombinant VP2 (rVP2) protein of CPV and explores their diagnostic potential for CPV detection.

\section{Materials and methods}

\section{Materials}

All reagents and chemicals used were of either analytical grade or HPLC grade. Recombinant VP2 protein was provided by TRPVB, TANUVAS Chennai. Tetra chloroauric acid was purchased from Sigma-Aldrich, India, and all membranes were purchased from MDI, India. TMB (tetramethyl benzidine), $\mathrm{H}_{2} \mathrm{O}_{2}$ (hydrogen peroxide), and DAB (di-amino benzidine) were purchased from Sisco Research Laboratories, India. Random N-30 ssDNA library and all primers were synthesized from Trilink Biotechnologies, USA. Monarch PCR and DNA clean up kit (5 $\mu \mathrm{g})$ and monarch DNA gel extraction kit were purchased from New England Biolabs, India. Hot start Taq polymerase was procured from Thermo Fisher Scientific. Streptavidin-coated magnetic beads (MMI-105 BcMagTM) were procured from Bioclone Inc., USA. A ssDNA library with 30 nucleotide central random region flanked with 23 nucleotide long constant primer binding region and having $10^{13}$ random sequences was used in the study. [5'TAG GGA AGA GGA CAT ATG AT (N30) TTG ACT AGT ACA TGA CCA CTT GA 3’]. Forward [5' TAG GGA AGA GAA GGA CAT ATG AT 3'] and reverse selection primer [5' TCA AGT GGT CAT GTA CTA GTC AA $3^{\prime}$ ] were synthesized without any modifications as well as with biotin modifications at 5' end. All other oligonucleotides used in the study were synthesized from GCC Biotech, India, and Eurofin, India. New generation sequencing of PCR amplicons was done by Clevergene Biocorp Pvt. Ltd., India. 


\section{Preparation of target antigen}

An earlier adapted field strain of CPV-2a was employed for the virus propagation (NCBI accession number $=\mathrm{KJ} 364524)$. The CPV was grown in bulk using A-72 cell line, and its titration and purification was carried out using previously described method with slight modifications (Teramoto et al. 1984; Verma et al. 2016). The cell culture grown virus was subjected to three cycles of freeze and thaw and then spun at $10,000 \mathrm{~g} \times 30 \mathrm{~min}$ at $4{ }^{\circ} \mathrm{C}$ to settle down all the cellular debris. The supernatant was adjusted to $0.4 \mathrm{M} \mathrm{NaCl}$ and precipitated with $8 \%$ PEG-6000 at $4{ }^{\circ} \mathrm{C}$ for overnight. The precipitated virion was allowed to settle at $10,000 \mathrm{~g} \times 30 \mathrm{~min}$ at $4{ }^{\circ} \mathrm{C}$, and the pellet was dissolved in TEN buffer (0.05 M Tris, $0.001 \mathrm{M}$ EDTA, $0.15 \mathrm{M} \mathrm{NaCl}, \mathrm{pH}$

7.4). Consequently, this was added to a gradient of sucrose (40\% and 60\%), and ultracentrifugation was carried out at $100,000 \mathrm{~g} \times 2.5 \mathrm{~h}$ at $4{ }^{\circ} \mathrm{C}$. The interface was carefully collected and allowed to centrifuge at 250,000 $g \times 2.15 \mathrm{~h}$ at $4{ }^{\circ} \mathrm{C}$ for final pelleting of virion. The pellet was dissolved in PBS and stored at $-80^{\circ} \mathrm{C}$. The reactivity of purified virion was confirmed using hemagglutination (HA) test and CPV immunochromatographic kit (Bionote). The concentration of purified virion was determined by Micro BCA protein assay kit (Pierce, USA). Additionally, rVP2 protein of CPV in the form of virus-like particles (VLPs) was kindly provided by TRPVB, TANUVAS Chennai, India, for use as target antigen in SELEX procedure. Earlier, this protein was also used by TRPVB, TANUVAS Chennai, India, for seroconversion and safety study by immunizing target species (dogs) under a separate grant funded by Biotechnology Industry Research Assistance Council (BIRAC) India (Scheme code: Ref. No.BT/CRS0156/CRS -07/15. Upon receiving rVP2, its functional activity was further evaluated at IVRI, Izatnagar, India, by HA and hemagglutination inhibition (HI) test before being utilized for SELEX.

\section{Membrane SELEX procedure}

Membrane SELEX procedure was adopted from previously described report with slight modifications (Tripathi et al. 2020). Membrane SELEX was accomplished using treated $0.2-\mu \mathrm{m}$ pore size nitrocellulose membrane and VP2 antigen as target. Prior to use, nitrocellulose membranes (pore size of $0.45 \mu \mathrm{m}$ ) were prepared following the protocol described previously by sequential immersion and washing in $0.5 \mathrm{M}$ $\mathrm{KOH}$, followed by $0.1 \mathrm{M}$ Tris base, $\mathrm{pH} 7.4$, and finally rinsed with HMCKN buffer (Frith et al. 2018). Ten microliter $(100 \mathrm{nmol})$ ssDNA library was denatured at $95^{\circ} \mathrm{C}$, for $10 \mathrm{~min}$, kept on ice immediately for $5 \mathrm{~min}$, and brought at RT for $15 \mathrm{~min}$. To this, $30 \mu \mathrm{L}$ of rVP2 protein $(0.45 \mathrm{mg} /$ $\mathrm{mL}$ ) and $400 \mu \mathrm{L}$ of binding buffer (PBS $1 \times$, herring sperm DNA $0.1 \mathrm{mg} / \mathrm{mL}$, and $10 \mathrm{mM} \mathrm{MgCl}_{2}$ ) were added and incubated for $2 \mathrm{~h}$ to allow binding of rVP2 with random ssDNA library. Following incubation, target-library mixtures were then passed through nitrocellulose membranes (positive selection), which were then rinsed by passing HMCKN buffer to remove all unbound sequences. It was assumed that all protein/target aptamer complexes were retained on the nitrocellulose filter. The retained ssDNA target complexes were eluted using $100 \mu \mathrm{L}$ elution buffer ( $7 \mathrm{M}$ urea, $100 \mathrm{mM}$ citrate buffer, and $3 \mathrm{mM}$ EDTA, $\mathrm{pH}$ 8.0) and precipitated via phenol-chloroform extraction method. Obtained sequences were PCR amplified using forward and biotinylated reverse primers, and the amplified product was visualized on $2.5 \%$ agarose gel. The first SELEX round PCR amplicon was eluted using gel extraction kit following manufacturer's protocol (Qiagen, USA). The eluted product was then subjected to strand displacement using streptavidin-coated magnetic beads (Bioclone Inc., USA) to retrieve biotinylated strand. The biotinylated strand was then subjected to second round of membrane SELEX, and the iterative process was repeated for seven rounds out of which two rounds were counter selection with fecal matrix and SPG cell lysate. Stringency was increased with every subsequent SELEX cycle by reducing the protein concentration and nucleotide-VP2 incubation time (Table S1, supplementary information). The last round PCR amplicon was then sent for new generation sequencing.

\section{Aptamer-VP2 binding analysis using in silico tools}

NGS analysis provided various clusters of repetitive sequences where each cluster had a representative sequence called seed sequence. We moved ahead with ten seed sequences selected from top 10 clusters from NGS data on the basis of their unique reads and number of times the sequences represented. The 10 aptamer sequences were named as A1-A10 for convenience. The secondary structure of all 10 aptamer sequences was predicted using M-fold server to analyze the stability profiling and loop resemblances between various aptamers. The secondary structures were modeled at default parameters consisting of $25{ }^{\circ} \mathrm{C}$ temperature, $5 \%$ sub-optimality number, limit of 50 for upper bound computing and $1 \mathrm{M}[\mathrm{NA}+]$ (Rouillard et al. 2003). The modeling of VP2 protein was accomplished using modweb (the online version of modeler) (Fiser and Sali 2003), and the modeled structure after the energy minimization step on SPDBV was further validated for analysis of Ramachandran plot (Lovell et al. 2002), ERRAT2 quality factor (Colovos and Yeates 1993), and Z score on PROSA (Wiederstein and Sippl 2007). The modeling of tertiary structures of ssDNA was done by mutating the structures of corresponding RNA construct. The RNA composer web server (Antczak et al. 2016) and Discovery studio visualizer (Dassault Systèmes 2017) were used to mutate and analyze their tertiary structure. The docking of modeled tertiary 
structure of ssDNA and modeled VP2 was performed with help of the Patchdock at a lustering RMSD of 4.0 (Schneidman et al. 2005), and target-ligand interactions were predicted using Ligplot (Schneidman et al. 2005).

\section{Aptamer-VP2 binding analysis using in vitro assays}

All 10 aptamer sequences were analyzed for their binding first with $\mathrm{rVP} 2$ protein and then with CPV using dot blot assay and enzyme-linked oligonucleotide assay (ELONA) as described below. In all assays, appropriate negative control (two random biotin labeled DNA sequences targeting cytochrome $\mathrm{b}$ and COX 1 gene of buffalo and pig, respectively) and positive control (CPV-specific dog serum) were employed.

Dot blot: Dot blot was performed as per the method described previously with slight modifications (Sypabekova et al. 2017). Briefly, $2 \mu \mathrm{L}$ rVP2 protein or $2 \mu \mathrm{L}$ purified $\mathrm{CPV}$ was immobilized on nitrocellulose (NC) membrane and incubated at room temperature for $1 \mathrm{~h}$. The membrane was washed in tris borate saline (TBS) buffer, $\mathrm{pH} 7.4$ followed by blocking with $5 \%$ casein for $60 \mathrm{~min}$ at room temperature. After washing with PBS, $5 \mu \mathrm{L}$ biotin-modified aptamers (previously exposed to $85^{\circ} \mathrm{C}$ for $5 \mathrm{~min}$ followed by incubation at room temperature for $10 \mathrm{~min}$ and at $37^{\circ} \mathrm{C}$ for $15 \mathrm{~min}$ ) applied on the membrane and incubated at $37^{\circ} \mathrm{C}$ for $45 \mathrm{~min}$. After completion of incubation and washing thrice with PBST, $5 \mu \mathrm{L}$ of Streptavidin-HRP conjugate (1:2500) was added and incubated for $45 \mathrm{~min}$ at $37^{\circ} \mathrm{C}$. With further washing, $\mathrm{DAB} / \mathrm{H}_{2} \mathrm{O}_{2}$ substrate was added. Appearance of brown dot on test versus no spot on control was considered as positive reaction. The positive control was put using dog anti-CPV serum in place of aptamer, and this was probed with biotinylated anti-dog IgG. Biotin labeled sequences targeting cytochrome b and COX 1 gene of buffalo and pig, respectively, were used as the negative control in place of aptamer.

ELONA: The binding of aptamers with purified CPV was further investigated by enzyme-linked oligonucleotide assay (ELONA) as per the method described previously with slight modifications (Xu et al. 2017). Briefly, $100 \mu \mathrm{L}$ of $500 \mathrm{ng}$ purified virus diluted in carbonate buffer, $\mathrm{pH}$ 9.6, was coated on the 96-well plate following incubation at $4{ }^{\circ} \mathrm{C}$ for overnight period. The plates were washed twice at an interval of 2 min with TBS buffer, $\mathrm{pH} 7.4$, to remove unbound or loosely bound virus particles. The non-specific binding sites on microtiter plate wells were blocked by incubating the plates with $200 \mu \mathrm{L}$ of $5 \%$ casein at room temperature for $1 \mathrm{~h}$. After washing the plates twice with PBS, pH 7.4, $100 \mu \mathrm{L}$ biotinylated aptamers (1A-10A) at concentration of $500 \mathrm{nM} /$ well were added to the plates and allowed to interact with immobilized virus particles by incubating at $37^{\circ} \mathrm{C}$ for $45 \mathrm{~min}$. All aptamer sequences were allowed to unfold to single strand and adopt its final confirmation by exposing to $85{ }^{\circ} \mathrm{C}$ for $5 \mathrm{~min}$ followed by incubation at room temperature for $10 \mathrm{~min}$ and then at $37{ }^{\circ} \mathrm{C}$ for $15 \mathrm{~min}$. The plates were washed thrice with PBST to remove unbound aptamer sequences from the wells. This was followed by addition of $100 \mu \mathrm{L}$ of Streptavidin-HRP conjugate (1:4000) per well which can noncovalently interact with biotinylated aptamers on plate surface. After completion of incubation at $37^{\circ} \mathrm{C}$ for $45 \mathrm{~min}$, unbound Streptavidin-HRP conjugate was eliminated by four times washing with PBST. TMB/ $\mathrm{H}_{2} \mathrm{O}_{2}(50 \mu \mathrm{L} /$ well $)$ was added to the wells and incubated at RT for $15 \mathrm{~min}$ in dark for catalytic oxidation of substrate by anchored HRP on the wells. The enzyme substrate reaction was terminated after 15 min with addition of $50 \mu \mathrm{L}$ of $1 \mathrm{M} \mathrm{H}_{2} \mathrm{SO}_{4}$ to each well. The absorbance (OD) was measured at $450 \mathrm{~nm}$ with the help of microplate ELISA reader (Thermo, USA).

\section{Determination of affinity or dissociation equilibrium constant (Kd)}

On the basis of the binding analysis results in different in vitro assays, four aptamer sequences $5 \mathrm{~A}, 6 \mathrm{~A}, 9 \mathrm{~A}$, and $10 \mathrm{~A}$ were selected to determine their binding affinity with rVP2 as well as CPV. Dissociation constant of the aptamer sequences was determined with rVP2 protein by coating the protein on NC membrane (Tripathi et al. 2020). Varying concentrations of aptamers $(7 \mathrm{nM}-2 \mu \mathrm{M})$ were added on respective membranes to allow incubation with immobilized rVP2. After washing, the bound sequences were eluted from the membrane and precipitated, and their concentration was determined with NanoDrop. Further, the dissociation constant $(\mathrm{Kd})$ of these aptamers was also determined with CPV using ELONA protocol as described previously by plotting the reading of bound sequences (after deducting blank reading) versus aptamer concentration $(7 \mathrm{nM}-2 \mu \mathrm{M})$ on GraphPad prism software (Xu et al. 2017). The analysis was done by plotting one site-specific saturation binding analysis.

\section{Determination of specificity of selected aptamers}

The specificity or cross-reactivity of all four selected aptamers was determined by ELONA as described above using different canine enteric viruses and purified dog $\operatorname{IgG}$ or phosphate buffered saline. The canine enteric viruses such as canine parvovirus $2 \mathrm{a}$, canine adenovirus-2 (CAV2 ), canine distemper virus (CDV), and canine corona virus $(\mathrm{CCoV})$ were kindly provided by the project monitoring unit, TANUVAS, Chennai, India. 


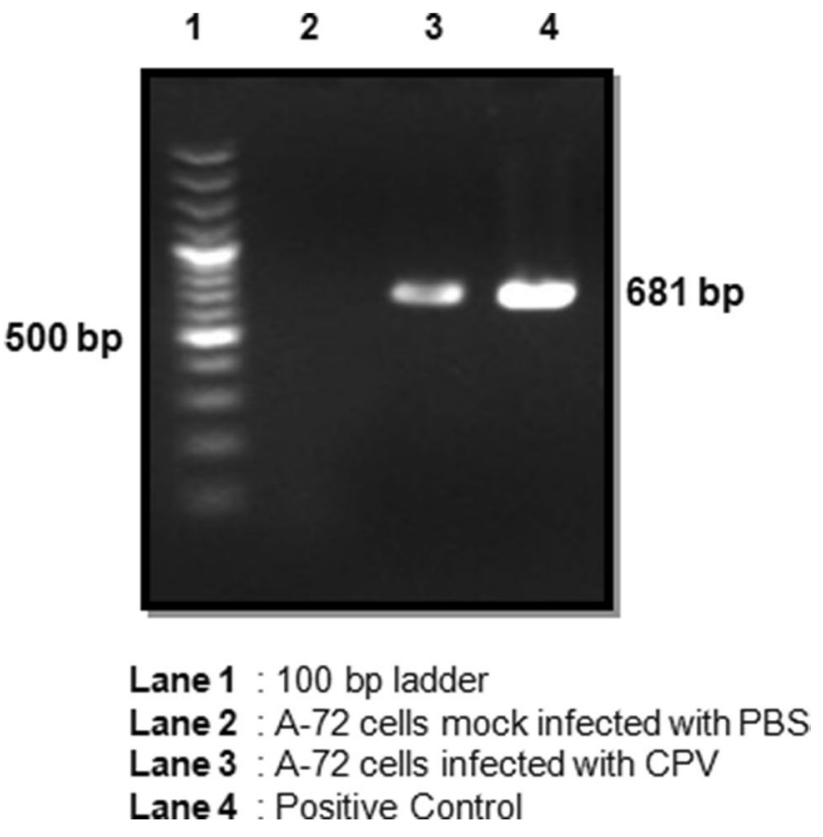

Fig. 1 Gel electrophoresis image of PCR amplicon depicting a $641 \mathrm{bp}$ band confirming the purity of prepared virion particles

\section{Statistical analysis}

The graphs for dissociation constants and specificity were plotted using graph pad prism software version 7.0. Statistical analysis of ELONA results was done using one-way ANOVA, and the data of cross-reactivity was analyzed using two-way ANOVA. Error bar indicates standard deviation, and $p$ value less than 0.05 was considered statistically significant.

\section{Results}

\section{Preparation of target antigen}

Virus growth in A-72 cells was characterized by rounding and shortening of cells in comparison to mock infected cells. The PCR (Fig. 1) and hemagglutination results (Fig. 2a) revealed a characteristic 681 bp product and HA titers of 512 (HA titers $\geq 32$ positive for $\mathrm{CPV}$ ), respectively, indicating the presence of virus. The titer of cell culture grown CPV was determined as $10^{4.3} \mathrm{TCID}_{50} / \mathrm{mL}$. The purified virion displayed strong hemagglutination titers of about 20,480 and
Fig. 2 (a) HA test for CPV, row $1-2=$ cell culture grown virus showing haemagglutination up to 9 th well $($ HA titer $=512)$ characterized by matt formation, row $3=\mathrm{RBC}$ control characterized by button formation; (b) immunochromatographic test for purified CPV; appearance of intense line at T-region indicating the intactness of purified virus following purification; (c) virus-like particles showing hemagglutination of porcine RBCs, row $1-2=$ virus-like particles (VP2 protein) (1:10 dilution) showing haemagglutination up to 11th well (HA titer $=2048$ ) characterized by matt formation, row $3=\mathrm{RBC}$ control characterized by button formation

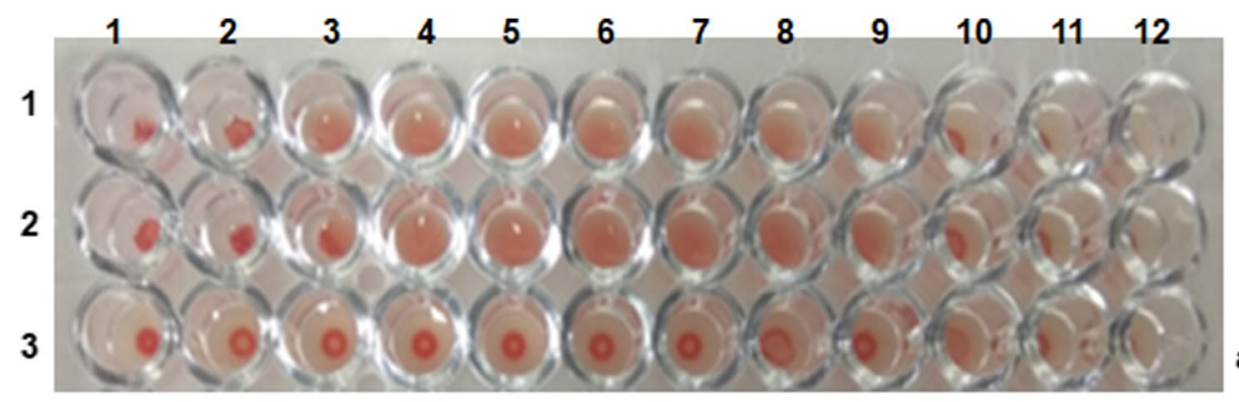

a)

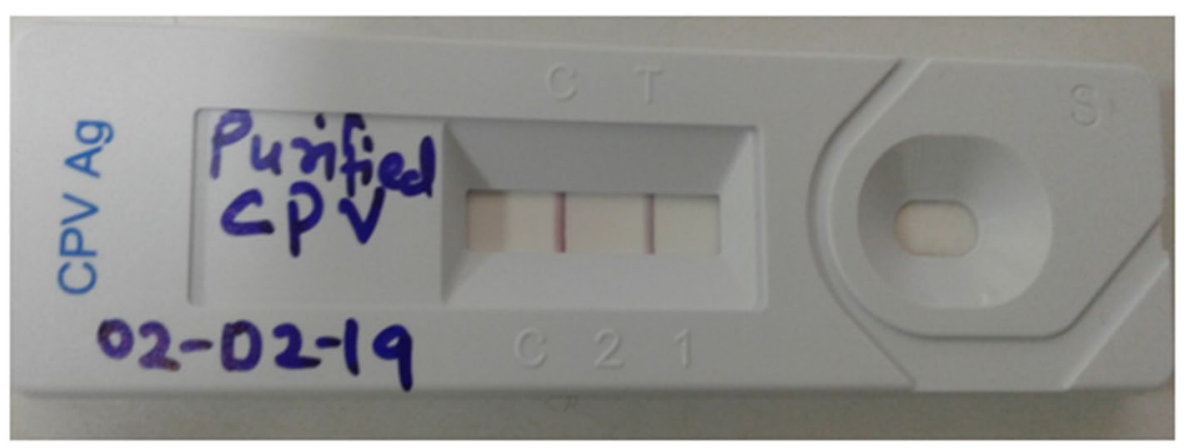

b)

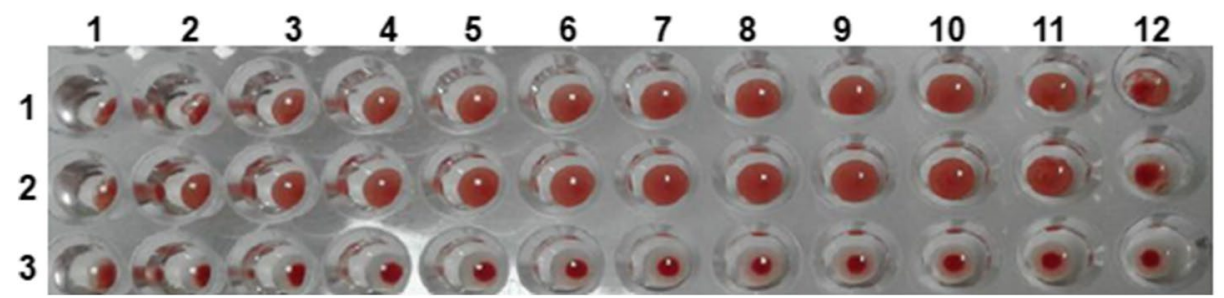

c) 
fair reactivity in commercial CPV immunochromatographic kit (Bionote) (Fig. 2b). The concentration of purified virion was estimated as $720 \mu \mathrm{g} / \mathrm{mL}$.

The hemagglutination titer of VLPs (rVP2) was recorded as 20,480 (Fig. 2c). The specificity of VLPs was confirmed by $\mathrm{HI}$ test using known CPV-positive dog serum which significantly inhibited the hemagglutination of porcine RBCs caused by VLPs.

\section{In silico characterization of aptamers against VP2}

A $76 \mathrm{bp}$ product was obtained at the last round of SELEX (Fig. S1, supplementary information) and was sent for NGS analysis. The NGS data set was utilized to characterize in silico all tentative aptamer candidates using multiple computational tools of repute. The secondary structure of aptamers was deduced through the $\mathrm{M}$-fold web server $25{ }^{\circ} \mathrm{C}$ to analyze the stability profiling and loop resemblances between various aptamers. The predicted secondary structures of the A1-A10 aptamers are presented in Fig. S2, supplementary information, and their corresponding $\Delta \mathrm{G}$ values are presented in Table 2 . The modeling of VP2 protein is accomplished using modweb (the online version of modeler), and the tertiary structure of the macromolecule after energy minimization is shown in Fig. 3a. Upon validation of the structure using Ramachandran plot showed the presence of only $0.4 \%$ of amino acids in disallowed region, ERRAT2 analysis resulted into a significant quality factor of 76.907 , and PRO-SA resulted into a significant $\mathrm{z}$ score of -6.42 . The modeling of tertiary structures of ssDNA was done by mutating the structures of corresponding RNA construct, and Fig. 3b depicts the tertiary structure of an exemplary aptamer candidate. Binding analysis of rVP2 with tertiary structure of all ten aptamers was performed using patch dock server. Figure $3 c$ depicts a representative image of the rVP2aptamer docked complex. The algorithm of Patchdock server directly relates the binding score with affinity of interaction; i.e., the higher the binding score, the stronger would be the binding between interacting molecules. Table 1 enlists the binding scores of all aptamers with rVP2 as predicted with patch dock. The binding score was obtained in the following order $6 \mathrm{~A}>9 \mathrm{~A}>5 \mathrm{~A}>10 \mathrm{~A}>3 \mathrm{~A}>2 \mathrm{~A}>7 \mathrm{~A}>1 \mathrm{~A}>8 \mathrm{~A}>$ 4A. 6A, 9A, 5A, and 10A emerged as the top four aptamer sequences showing higher binding strength with rVP2. The
Fig. 3 (a) 3D structure of recombinant VP2 modeled using modweb, (b) tertiary structure of $6 \mathrm{~A}$ aptamer, and (c) a representative aptamer-rVP2 protein docking complex using patch dock server
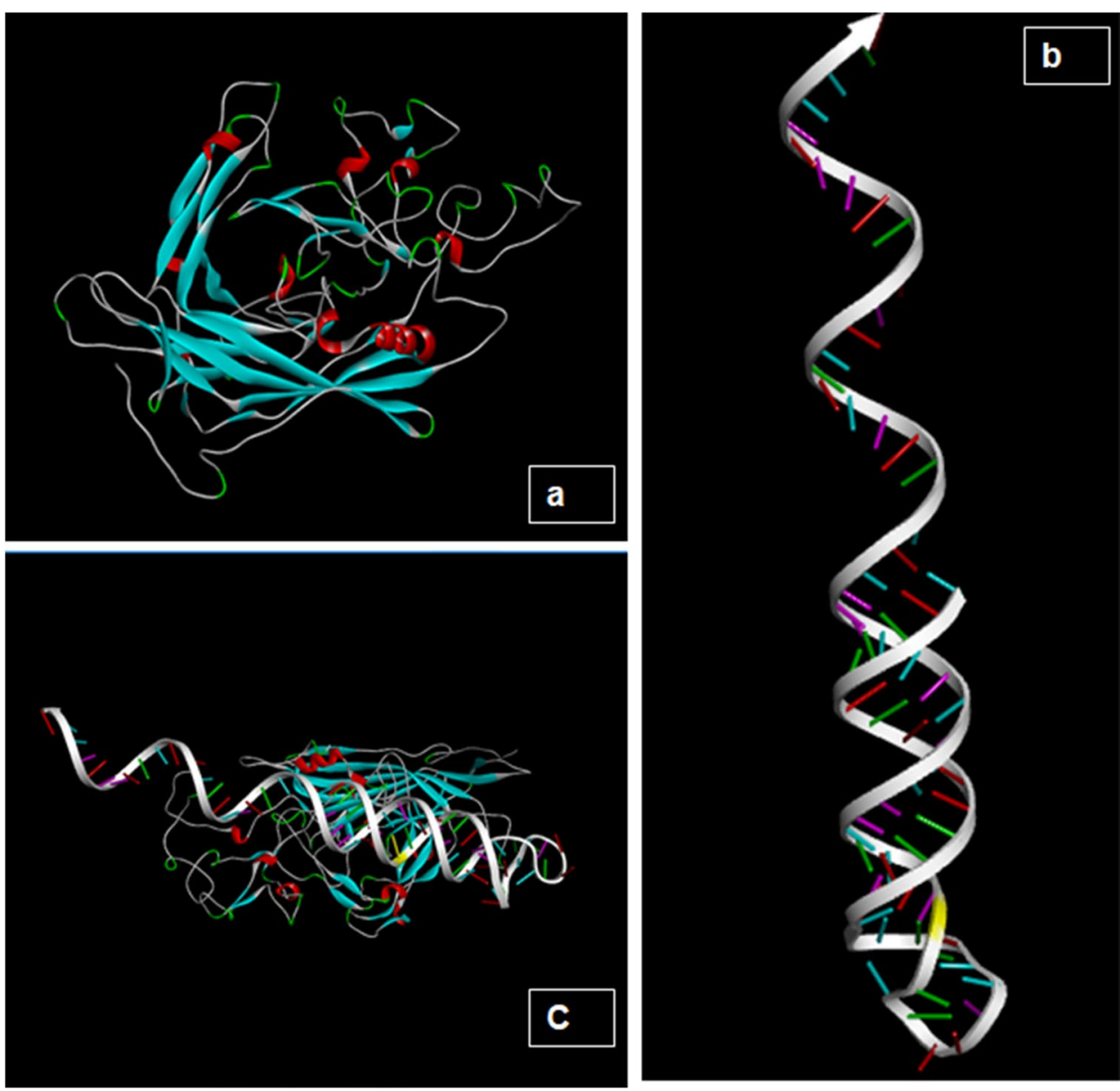
Table $1 \Delta \mathrm{G}$ values of secondary structures of A1-A10 aptamers and the binding score of aptamer docking with rVP2 as predicted with patch dock

\begin{tabular}{lll}
\hline Aptamer ID & $\begin{array}{l}\Delta \text { G values of secondary } \\
\text { structures }\end{array}$ & $\begin{array}{l}\text { Binding score of } \\
\text { docked com- } \\
\text { plexes }\end{array}$ \\
\hline 1A & -26.81 & 20,812 \\
$2 A$ & -28.71 & 21,632 \\
$3 A$ & -5.68 & 21,896 \\
$4 A$ & -15.55 & 19,990 \\
$\mathbf{5 A}$ & -19.88 & 23,830 \\
$\mathbf{6 A}$ & -28.48 & 28,686 \\
$7 \mathrm{~A}$ & -6.31 & 21,384 \\
$8 \mathrm{~A}$ & -22.23 & 20,752 \\
$\mathbf{9 A}$ & -30.38 & 23,992 \\
$\mathbf{1 0 A}$ & -17.00 & 23,362 \\
\hline
\end{tabular}

residues of aptamers interacting with $\mathrm{rVP} 2$ are analyzed and enlisted in Table S2, supplementary information.

\section{Aptamer-VP2 binding analysis using in vitro assays}

Dot blot: The qualitative binding of the biotinylated aptamers (A1-A10) with rVP2 was analyzed with dot blot assay. Figure 4a depicts the blot images showing a dark spot which is indicative of positive reaction of aptamer sequences with immobilized rVP2 protein. Aptamer 7A and $8 \mathrm{~A}$ also interacted with $\mathrm{rVP} 2$ as evident from the red color spot of captured gold nanoparticle labeled rVP2, though its negative control had a faint spot reflecting some background signal. The negative control in other blots lacks any spot, and positive control was put using CPVspecific dog serum in place of aptamer. In order to further confirm the binding of aptamers with cultured virus particles, dot blot was performed with CPV. Figure $4 \mathrm{~b}$ depicts the results of CPV binding with all aptamers. These results indicate that though all aptamers were able to bind with
Fig. 4 Dot blot images of aptamers (1A-10A). (a) Blots showing interaction of aptamers with immobilized rVP2 at $500 \mathrm{nM}$ aptamer concentration, the blots of 7A and 8A were carried out using gold nanoparticle labeled rVP2; (b) blots showing interaction of aptamers with immobilized CPV at $1 \mu \mathrm{M}$ concentration. "Positive" control: known dog anti-CPV serum in place of aptamer probed with anti-dog IgG biotin label; "Negative" control: biotin labeled sequences targeting cytochrome $b$ and COX 1 gene of buffalo and pig respectively in place of aptamer
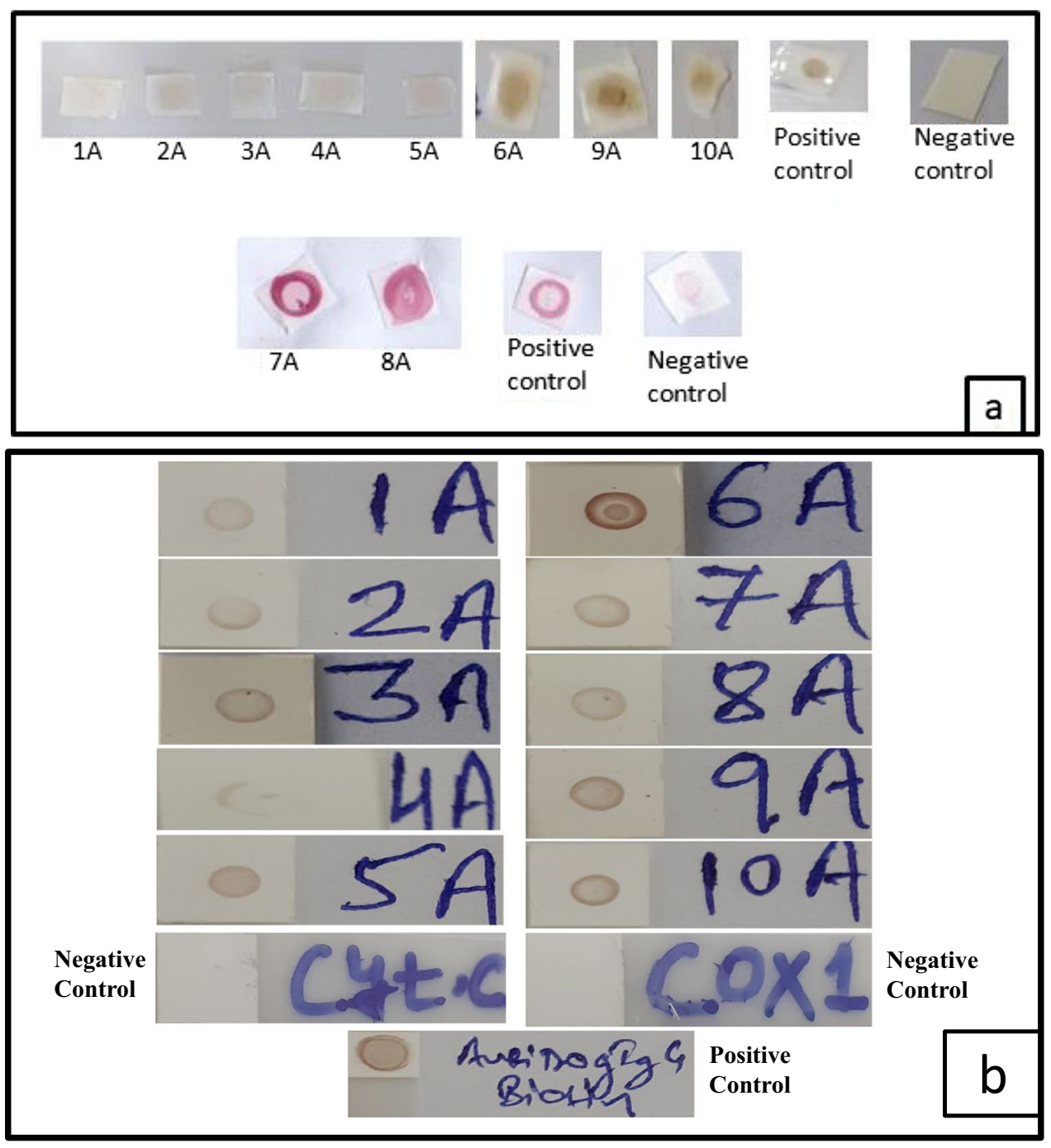


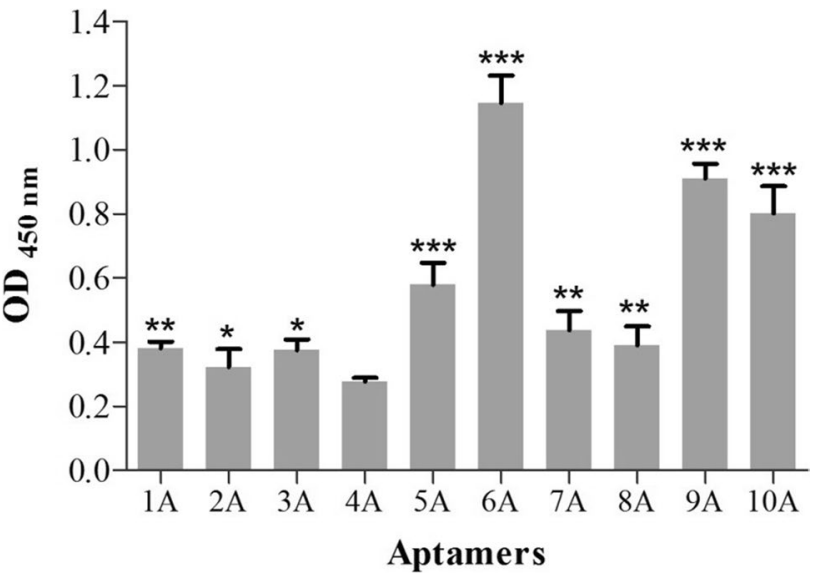

Fig. 5 Graph depicting relative binding abilities of screened aptamers $(500 \mathrm{~nm})$ with CPV through ELONA. The data was analyzed with one way ANOVA $(*<0.05 ; * *<0.01 ; * * *<0.001)$

rVP2 or CPV, but aptamers 5A, 6A, 9A, and 10A produced relatively more intense spots.

ELONA: The binding of aptamers with CPV was further investigated at $500 \mathrm{nM}$ aptamer concentration in ELONA. Figure 5 depicts relative binding of the aptamers with CPV. The data is corrected for values of negative control. It is evident that all aptamers were able to recognize the CPV target, but 5A, 6A, 9A, and 10A emerged as top four sequences that bind CPV with high reactivity. The data was found significant with respect to control $(p<0.05)$ except $4 \mathrm{~A}$ aptamer. It is observed that aptamer 6A displayed significantly high binding signal in both ELONA and dot blot as compared to other three aptamers: $5 \mathrm{~A}, 9 \mathrm{~A}$, and $10 \mathrm{~A}(p<0.001)$.

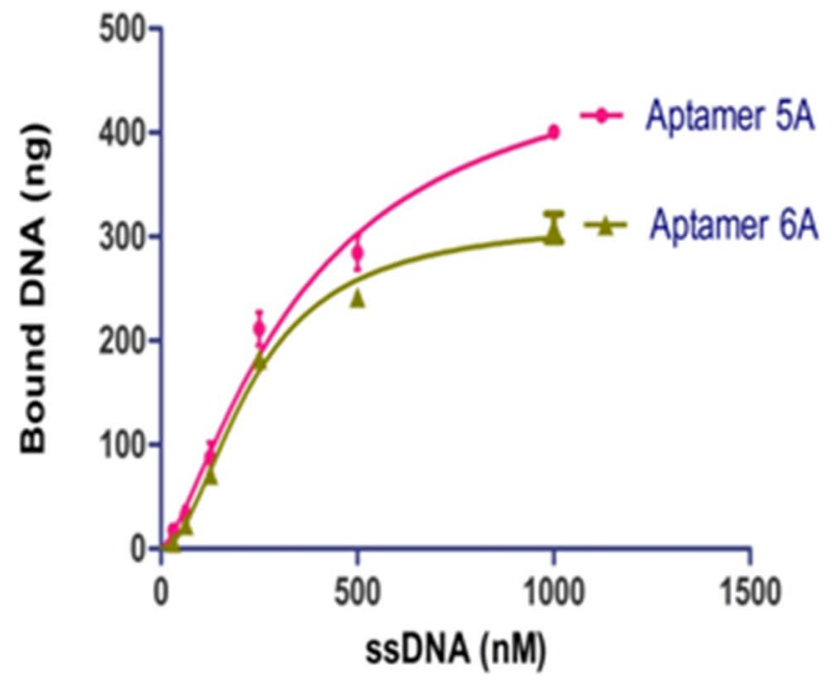

\section{Dissociation constant determination}

The dissociation constant $(\mathrm{Kd})$ of $5 \mathrm{~A}, 6 \mathrm{~A}, 9 \mathrm{~A}$, and 10A was determined with rVP2 as well as CPV. Figure 6 depicts the saturation binding analysis graphs of aptamers at their various concentrations against rVP2 protein. The saturation binding graphs are also plotted for specific binding of aptamers with CPV as depicted in Fig. 7.

The Kd determination using these plots by GraphPad prism is enlisted in Table 2. It shows that the dissociation constant of $6 \mathrm{~A}$ aptamer was lowest $230.6 \mathrm{nM}$ with rVP2 and $105.1 \mathrm{nM}$ with CPV, respectively. $6 \mathrm{~A}$ and $10 \mathrm{~A}$ aptamers were found to bind the CPV with 4-5 times high affinity and hence could be considered as better binding aptamers.

\section{Specificity of the aptamers}

The specificity of four aptamers 5A, 6A, 9A, and 10A with different viruses is depicted in Fig. 8 by plotting the PBS value as negative control. All aptamers displayed specific reactivity with CPV (CPV2a), while 5A, 9A, and $10 \mathrm{~A}$ displayed low binding with other canine enteric viruses such as CAV-2, CDV, and $\mathrm{CCoV}$ as conformed by ELONA. On the contrary, 6A showed high cross-reacting signal with CCoV and CDV viruses. Dog IgG was also tested for any cross-reactivity with selected aptamers; however, no significant binding was seen with any of these aptamers. The results were found to be highly significant $(p-<0.0001)$. Dot blot results using standard $\mathrm{CPV}$ positive dog serum also resulted into similar findings. Additionally, with any of the selected aptamers, no cross-reactivity was observed with either dog $\operatorname{IgG}$ or PBS as conformed by dot blot and ELONA results.

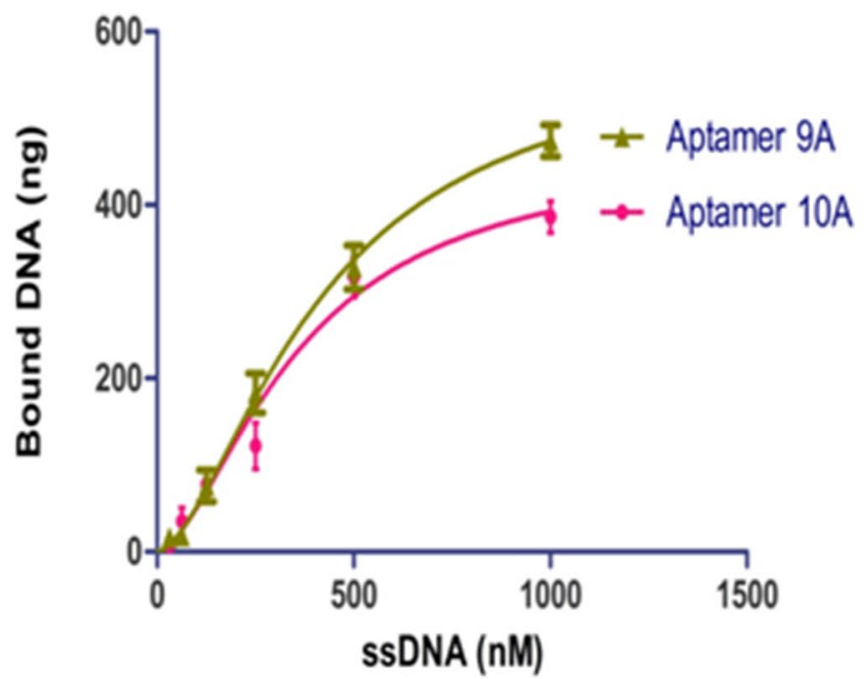

Fig. 6 Saturation binding analysis graphs of aptamers with rVP2 for Kd determination 


\section{A Aptamer}

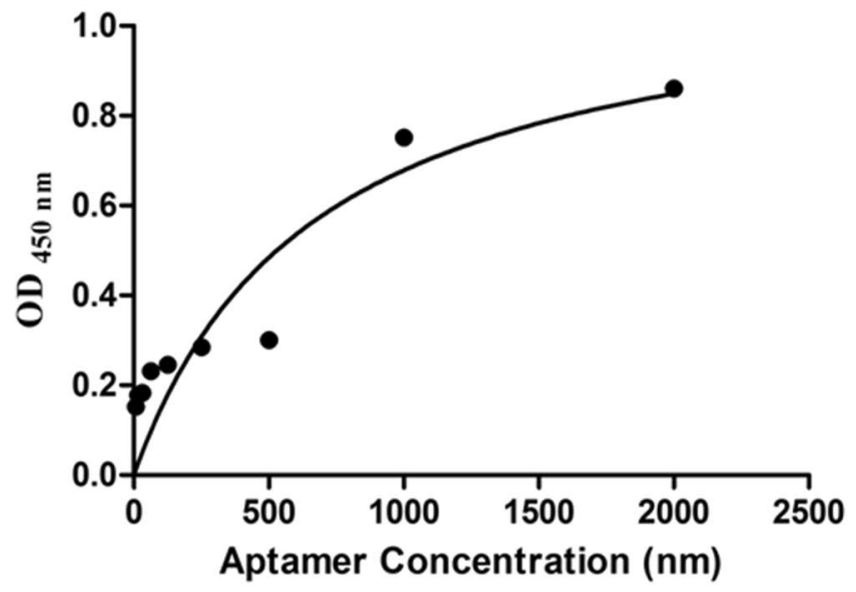

9A Aptamer

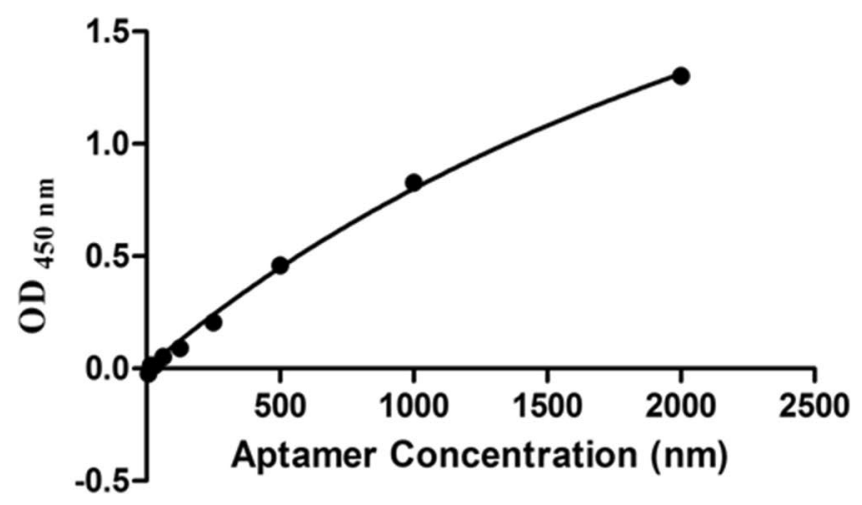

6A Aptamer

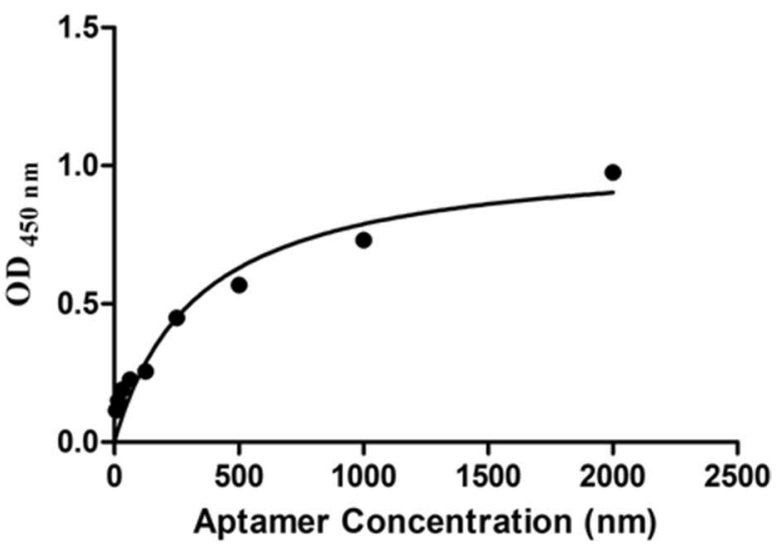

10A Aptamer

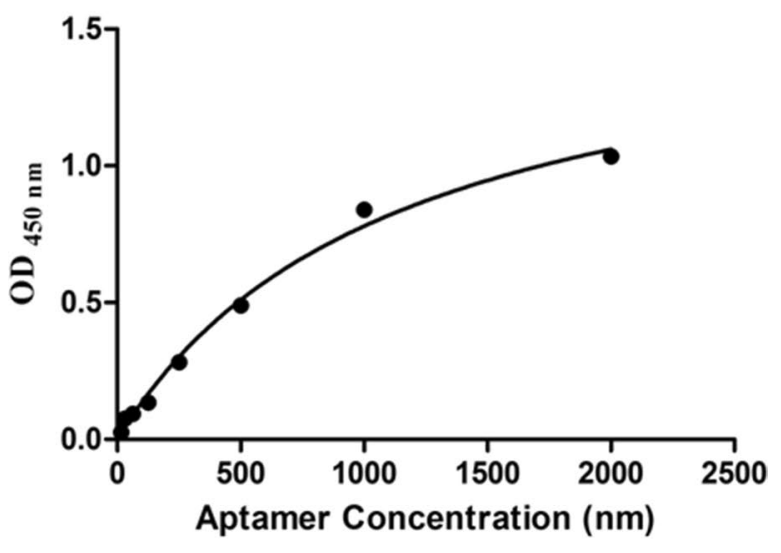

Fig. 7 Saturation binding analysis graphs of aptamers with CPV for Kd determination

Table 2 Dissociation constants of screened aptamers (5A, 6A, 9A, 10A) with rVP2 and CPV

\begin{tabular}{|c|c|c|c|}
\hline Aptamer ID & Central random sequence $\left(5^{\prime}-3^{\prime}\right)$ & $\begin{array}{l}\mathrm{Kd}(\mathrm{nM}) \\
\text { with } \\
\text { rVP2 }\end{array}$ & $\begin{array}{l}\text { Kd (nM) } \\
\text { with CPV }\end{array}$ \\
\hline $5 \mathrm{~A}$ & $\begin{array}{l}\text { TAGGGAAGAGAAGGACATATGATTCAAGTGGTCATGTCCTTCTCTTCCCTATTGACTAGTACA } \\
\text { TGACCACTTGA }\end{array}$ & 359.5 & 429.3 \\
\hline $6 \mathrm{~A}$ & $\begin{array}{l}\text { TAGGGAAGAGAAGGACATATGATAGTGGTCATGTACTAGTCAACGTACCCGGCACTAGCCCAG } \\
\text { CTGTCCGCCCTTGACTAGTACATGACCACTTGA }\end{array}$ & 230.6 & 105.1 \\
\hline $9 \mathrm{~A}$ & $\begin{array}{l}\text { TAGGGAAGAGAAGGACATATGATAAGTGGTCATGTACTAGTCAACGGGCCCGGGATCCGATTT } \\
\text { GACTAGTACATGACCACTTGA }\end{array}$ & 359.3 & 557.6 \\
\hline $10 \mathrm{~A}$ & $\begin{array}{l}\text { TAGGGAAGAGAAGGACATATGATATGTACTAGTCAACGCCCCCCGCCCTTGACTAGTACATGA } \\
\text { CCACTTGA }\end{array}$ & 416.3 & 164.8 \\
\hline
\end{tabular}

\section{Discussion}

Aptamers have emerged as specific and high binding affinity molecular probes having tremendous diagnostic and therapeutic potential. ssDNA aptamers are preferred over RNA aptamers to be used for any diagnostic applications as the former are more stable and robust.
Various SELEX strategies have been designed and explored to screen target-specific aptamers; however, membrane-based SELEX is considered an efficient and convenient SELEX strategy for identification of targetspecific aptamers (Bayat et al. 2018). We have used nitrocellulose membrane filtration SELEX for identification of ssDNA aptamers against rVP2 protein of 


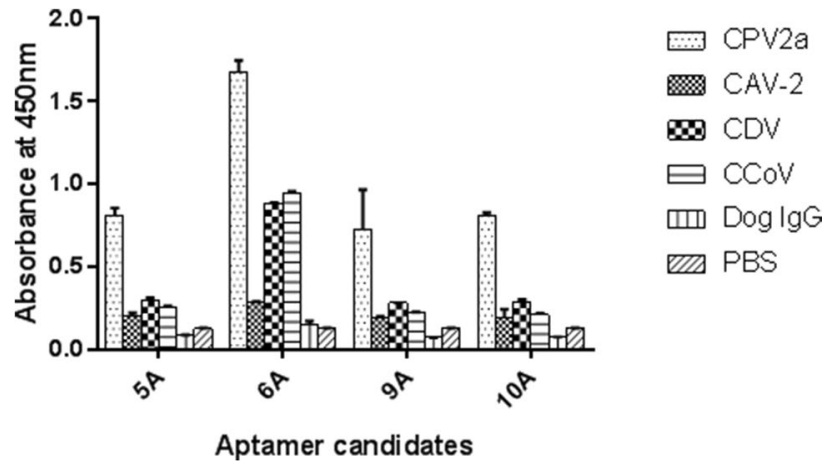

Fig. 8 Graph representing cross-reactivity of selected aptamers (5A, 6A, 9A, and 10A) with common canine enteric viruses using ELONA. The data was analyzed by two way ANOVA $(* * * *<0.0001)$

CPV. To prevent the non-specific binding of ssDNA on the membrane, it was treated with HMCKN buffer as described previously (Frith et al. 2018). To confirm, the HMCKN-treated membrane was incubated with random ssDNA for $2 \mathrm{~h}$. The membrane was washed and treated with elution buffer to elute any non-specifically bound sequences. The eluted product was precipitated, PCR amplified, and visualized on gel. The absence of band on the gel confirmed that HMCKN treatment prevented non-specific ssDNA binding on the membrane. In order to target all potential binding sites of the rVP2 protein, the ssDNA library was incubated with rVP2 in solution in 1:10 molar ratio in first SELEX round. The concentration of target protein (rVP2) and time of incubation was reduced with proceeding SELEX rounds so as to obtain only high affinity binding sequences (Table S1).

NGS analysis provided seed sequences of top ten aptamer clusters on the basis of their frequency reads which were used for their bioinformatics characterization. The binding score obtained after docking these aptamers (A1-10) with modeled rVP2 showed that $6 \mathrm{~A}$ aptamer binds to $\mathrm{rVP} 2$ with highest binding score $(28,686)$ followed by $9 \mathrm{~A}, 5 \mathrm{~A}$, and $10 \mathrm{~A}$ aptamers whose binding score was not distinctly different from each other (Table 1). It can be assumed that $6 \mathrm{~A}$ was interacting very strongly with modeled rVP2 compared to other aptamers, and this assumption was also supported by analyzing the number of hydrogen bonds/hydrophobic interactions in the docked complexes. Table S2, Supplementary information, depicts that $6 \mathrm{~A}$ formed maximum bonds with modeled rVP2 (28 H-bonds and 2 hydrophobic interactions), as compared to other aptamers, viz., 10A (12 H-bonds and 3 hydrophobic interactions), 9A (13 H-bonds and no hydrophobic interactions), and 5A (9 $\mathrm{H}$-bonds and 3 hydrophobic interactions). The binding residue analysis renders $6 \mathrm{~A}$ as the aptamer with most feasible binding efficacy. Moreover, each of the participating nucleotide in 6A aptamer forms $\mathrm{H}$-bond with many amino acids of rVP2 in parallel, whereas, for other aptamers, the nucleotides formed H-bonds with only one or two amino acid residues maximally. To validate the obtained in silico data, we analyzed the binding results of aptamers with rVP2 and CPV using dot blot as well as ELONA studies. Dot blot results also indicated that 6A, 9A, 10A, and 5A aptamers produced high-intensity color signals which correspond to their strong binding with rVP2 and CPV (Fig. 4a, b). A visibly highest intensity signal was seen in $6 \mathrm{~A}$ aptamer, whereas the color intensity of the dot for other three aptamers was visibly not very distinct. Since dot blot is a qualitative method, ELONA experiment was conducted to quantify the binding of aptamers with CPV. ELONA results were also in concordance indicating highest binding signal for 6A aptamer followed by 9A, $10 \mathrm{~A}$, and 5A (Fig. 5). From here, we predicted that 6A could recognize its target with high affinity. Hence, we determined the dissociation constant of these four aptamers with rVP2 as well as CPV. The Kd of 6A aptamer was also found to be the lowest with rVP2 $(230.6 \mathrm{nM})$ as well as CPV (105.1 nM) supporting in silico and other experimental findings.

The cross-reactivity experiments of all four aptamers with related viruses were quite interesting. As expected, $6 \mathrm{~A}$ aptamer was found to display maximum binding signal with CPV and negligible cross-reactivity with CAV-2 and $\operatorname{dog} \mathrm{IgG}$, whereas it showed almost $50 \%$ cross-reacting signal with $\mathrm{CDV}$ and $\mathrm{CCoV}$ viruses. On the other hand, 5A, 9A, and 10A aptamers had relatively low binding with CPV as compared to 6A aptamer, but the cross-reactivity of these three aptamers with other viruses was very low (Fig. 8). Considering the specificity results, it could be said that though 6A aptamer binds CPV with highest affinity, it might possess some residues that readily participates in bond formation with other related viruses. The in silico data also showed that the nucleotides of $6 \mathrm{~A}$ interact with many amino acids of rVP2 through $\mathrm{H}$-bonds strengthening the finding that it could non-specifically interact with other virus particles possibly through $\mathrm{H}$-bonds.

Upon further analyzing the secondary structures of these aptamers, it was observed that a big circular loop was common in all sequences indicating it as a minimal essential requirement to attain a stable structure as it did not participate in major bond formations (Fig. S2). Most of the interacting residues in 6A aptamer were located at its unique end terminal small stem-loop structure rich in $\mathrm{G}$ and $\mathrm{C}$ residues and which is contributing towards high binding/cross-reactivity of this aptamer (Fig. S2 and Table S2). Aptamers 9A and 10A displayed longer stems ending with very small loops of cytosine residues, but the potential binding motif is 
probably the longer stem as indicated by the presence of high number of interacting residues in this region. In 5A aptamer, very few residues participated in interaction with its target and that too were scattered along the two circular loops rather than its stem. On the basis of obtained in silico and in vitro results, it could be stated that $10 \mathrm{~A}$ aptamer emerges as a good candidate sequence for recognizing CPV in terms of its binding strength, dissociation constant, and good specificity.

To summarize, the present study first time reports ssDNA aptamers that specifically binds with CPV with affinity in nanomolar range. Our findings demonstrated the diagnostic potential of these aptamers and present $10 \mathrm{~A}$ as a prospective molecular probe in developing efficient diagnostics for CPV.

Supplementary Information The online version contains supplementary material available at https://doi.org/10.1007/s00253-021-11651-x.

Acknowledgements We are grateful to our respective director (IVRI and MNNITA) for providing the necessary facilities to carry out the work. We are pleased to acknowledge the Department of Biotechnology (DBT), India, for financial support (Project No. BT/ADV/Canine Health//TANUVAS//2017-18) to this work. We are thankful to TANUVAS, Chennai, the project monitoring unit (PMU) for providing recombinant VP2 protein and other viruses used for cross-reactivity studies. The DST-FIST facility at Department of Biotechnology, MNNITA, is gratefully acknowledged. We also acknowledge Dr. Rajeev Ranjan, senior scientist, ICAR-IVRI, for sharing two oligosequences which were used as negative control in present study.

Author contribution $\mathbf{M S}^{\mathbf{1}}$ was involved in conducting experiments related to this study at IVRI, Bareilly, while PT and SM at MNNIT Alalhabad. $\mathbf{M S}^{2}$ supported with the planning and execution of molecular biology-related work, while VC and UKD contributed towards result analysis and compilation. GKS was involved in manuscript writing and provided critical advice on the manuscript. RKS provided critical inputs during revision of the manuscript. SK helped towards providing recombinant VP2. KP supported towards aptamer crossreactivity experiment. $\mathbf{M S}^{\mathbf{1}}$ and $\mathbf{S N}$ were responsible for the overall planning, execution, and supervision of this work at IVRI, Barielly, and MNNIT Allahabad, respectively. Both $\mathbf{M S}^{\mathbf{1}}$ and $\mathbf{S N}$ also contributed in problem solving, writing, and editing of this manuscript. All authors read and approved the manuscript.

Funding This work was financially supported by Department of Biotechnology, India (Project No. BT/ADV/Canine Health// TANUVAS//2017-18).

Data availability (data transparency) All data reported in this manuscript is original and part of the DBT (India) funded project.

Code availability Not applicable.

\section{Declarations}

Ethics approval This article does not contain any studies with human participants or animals performed by any of the authors.

Conflict of interest/Competing interests The authors declare no competing interests.

\section{References}

Antczak M, Popenda M, Zok T,Sarzynska J, Ratajczak T, Tomczy KK, Adamiak RW, Szachniuk M (2016) New functionality of RNA composer: an application to shape the axis of miR160 precursor structure. Acta Biochim Pol 63(4): 737-744. https://doi.org/10. 18388/abp.2016_1329

Bayat P, Nosrati R, Alibolandi M, Rafatpanah H, Abnous K, Khedri M, Ramezani M (2018) SELEX methods on the road to protein targeting with nucleic acid aptamers. Biochimie 154:132-155. https://doi.org/10.1016/j.biochi.2018.09.001

Chander V, Chakravarti S, Gupta V, Nandi S, Singh M, Badasara SK, Sharma, C, Mittal M, Dandapat S, Gupta VK (2016) Multiplex amplification refractory mutation system PCR (ARMS-PCR) provides sequencing independent typing of canine parvovirus. Inf Gent Evol 46:59e64. https://doi.org/10.1016/j.meegid.2016. 10.024

Cheng J, Wang J, Liu Y, Wu Q, Wang Z (2018) Screening and identification of ssDNA aptamers against $\mathrm{HN}$ protein for detection of bovine parainfluenza virus type 3 antibodies in serum. Curr Pharma Biotech 19(11):896-901. https://doi.org/10.2174/13892 01019666181031154046

Colovos C, Yeates TO (1993) Verification of protein structures: patterns of non bonded atomic interactions. Protein Sci 2(9):15111519. https://doi.org/10.1002/pro.5560020916

Dassault Systèmes BIOVIA, (Discovery studio visualizer), (2559350), San Diego: Dassault Systèmes, (2017)

Decaro N, Buonavoglia C (2012) Canine parvovirus: a review of epidemiological and diagnostic aspects, with emphasis on type 2c. Vet Microbiol 155:1-12. https://doi.org/10.1016/j.vetmic.2011. 09.007

Eregowda CG, De U K, Singh M, Prasad H, Akhilesh, Sharma K, Roy choudhury P, Rajesh JB, Patra MK, Behera SK (2020). Assessment of certain biomarkers for predicting survival in response to treatment in dogs naturally infected with canine parvovirus. Microb Pathog 149: 104485. https://doi.org/10.1016/j.micpath. 2020.104485

Fiser A, Sali A (2003) ModLoop: automated modeling of loops in protein structures. Bioinformatics 19(18):2500-2501. https://doi. org/10.1093/bioinformatics/btg362

Frith KA, Fogel R, Goldring JPD, Krause RGE, Khati M, Hoppe H, Cromhout ME, Jiwaji M, Limson JL (2018) Towards development of aptamers that specifically bind to lactate dehydrogenase of Plasmodium falciparum through epitopic targeting. Malar J 17:191. https://doi.org/10.1186/s12936-018-2336-Z

Hmila I, Wong Phatcharachai M, Laamiri N, Aouini R, Marnissi B, Arbi M, Sreevatsan S, Ghram A (2017) A novel method for detection of H9N2 influenza viruses by an aptamer-real time-PCR. J Virol Methods 24(3):83-91. https://doi.org/10.1016/j.jviromet. 2017.01.024

Joshi R, Janagama H, Dwivedi HP, Kumar TMAS, Jaykus LA, Schefers J, Sreevatsan S (2009) Selection, characterization, and application of DNA aptamers for the capture and detection of Salmonella enterica serovars. Mol Cell Probes 23:20e8. https://doi.org/10. 1016/j.mcp.2008.10.006

Kelman M, Barrs VR, Norris JM, Ward MP (2020) Canine parvovirus prevention and prevalence: veterinarian perceptions and behaviors. Prev Vet Med 174:104817. https://doi.org/10.1016/j.preve tmed.2019.104817

Liu G, Mao X, Phillips JA, Xu H, Tan W, Zeng L (2009) Aptamernanoparticle strip biosensor for sensitive detection of cancer cells. Anal Chem 81(24):10013-10018. https://doi.org/10.1021/ac901 $889 \mathrm{~s}$

Lovell SC, Davis IW, Arendall WB III, De Bakker PIW, Word JM, Prisant MG, Richardson JS, Richardson DC (2002) Structure 
validation by Calpha geometry: phi, psi and $\mathrm{C}$ beta deviation. Proteins 50:437-450. https://doi.org/10.1002/prot.10286

Marnissi B, Kamali-Moghaddam M, Ghram A, Hmilla I (2020) Generation of ssDNA aptamers as diagnostic tool for Newcastle avian virus. PLoS ONE 15(8):e0237253. https://doi.org/10.1371/journ al.pone. 0237253

Qi S, Zhao J, Guo D, Sun D (2020) A mini-review on the epidemiology of canine parvovirus in China. Front Vet Sci 7:1-10. https://doi. org/10.1016/j.prevetmed.2019.104817

Radi AE (2011) Electrochemical aptamer-based biosensors: recent advances and perspectives. Int J Electrochem 2011:1-17. https:// doi.org/10.4061/2011/863196

Rouillard JM, Zuker M, Gulari E (2003) OligoArray 2.0: design of oligonucleotide probes for DNA microarrays using a thermodynamic approach. Nucl Acids Res 31(12):3057-3062. https://doi. org/10.1093/nar/gkg426

Schneidman DD, Inbar Y, Nussinov R, Wolfson HJ (2005) PatchDock and SymmDock: servers for rigid and symmetric docking. Nucl Acids Res 33:W363-367. https://doi.org/10.1093/nar/gki481

Sharma C, Singh M, Upmanyu V, Chander V, Verma V, Chakrovarty S, Sharma GK, Dhanze H, Singh P, Shrivastava S, Kumar J, Goswami TK, Gupta VK (2018) Development and evaluation of a gold nanoparticle based immunochromatographic strip test for the detection of canine parvovirus. Arch Virol 163(9):2359-2368. https://doi.org/10.1007/s00705-018-3846-2

Sypabekova M, Bekmurzayeva A, Wang R, Li Y, Nogues C, Kanayeva D (2017) Selection, characterization, and application of DNA aptamers for detection of Mycobacterium tuberculosis secreted protein MPT64. Tuberculosis 104: 70-78. https://doi.org/10. 1016/j.tube.2017.03.004
Teramoto YA, Mildbrand MM, Carlson J, Collins JK, Winston S (1984) Comparison of enzyme-linked immunosorbent assay, DNA hybridization, hemagglutination and electron microscopy for detection of canine parvovirus infections. J Clin Microbiol 20(3):373-378. https://doi.org/10.1128/JCM.20.3.373-378.1984

Tombelli S, Minunni M, Mascini M (2007) Aptamers-based assays for diagnostics, environmental and food analysis. Biomol Eng 24(2):191-200. https://doi.org/10.1016/j.bioeng.2007.03.003

Torres-Chavolla E, Alocilja EC (2009) Aptasensors for detection of microbial and viral pathogens. Biosens Bioelectron 24(11):31753182. https://doi.org/10.1016/j.bios.2008.11.010

Tripathi P, Sachan M, Nara S (2020) Novel ssDNA ligand against ovarian cancer biomarker CA125 with promising diagnostic potential. Front Chem 8:400. https://doi.org/10.3389/fchem.2020.00400

Verma S, Singh M, Chander V, Glora P, Chakrovarty S, Thomas J, Goswami TK, Kumawat S (2016) Isolation of canine parvovirus-2 in A-72 cell Line. J Immunol Immunopathol 18(2):122-126. https:// doi.org/10.5958/0973-9149.2016.00021.6

Wiederstein M, Sippl MJ (2007) ProSA-web: interactive web service for the recognition of errors in three-dimensional structures of proteins. Nucleic Acids Res 35:W407-410. https://doi.org/10. 1093/nar/gkm290

Xu J, Zhang X, Zhou S, Shen J, Yang D, Wu J, Li X, Li M, Huang X, Sealy JE, Iqbal M, Li Y (2017) A DNA aptamer efficiently inhibits the infectivity of bovine herpesvirus 1 by blocking viral entry. Sci Rep 7:11796. https://doi.org/10.1038/s41598-017-10070-1

Publisher's note Springer Nature remains neutral with regard to jurisdictional claims in published maps and institutional affiliations. 